\title{
DIMENSÃO FRACTAL DE ÁCIDOS HÚMICOS EM DIFERENTES CONDIÇÕES EXPERIMENTAIS
}

\author{
Alessandro Costa da Silva e Eduardo Sá Mendonça \\ Departamento de Solos, Universidade Federal de Viçosa, 36571-000 Viçosa - MG \\ César Reis* \\ Departamento de Química, Universidade Federal de Viçosa, 36571-000 Viçosa -MG
}

Recebido em 24/7/02; aceito em 01/10/02

\begin{abstract}
FRACTAL DIMENSION OF HUMIC ACIDS IN DIFFERENTS EXPERIMENTAL CONDITION. The determination of fractal dimension (D) of humic particles was achieved by the turbidimetric technique where diluted suspensions of humic acids, in different experimental conditions, were analyzed by spectrophotometry UV-Vis. The slope of the lines $(\beta)$ was taken from the graphics $(\log \tau$ vs $\log \lambda)$ to obtain $\mathrm{D}$. The results show that the values of $\mathrm{D}$ changed according to $\mathrm{pH}(3.0,5.0$ and 7.0$)$, temperature (25 and $5{ }^{\circ} \mathrm{C}$ ) and shaking (magnetic and horizontal). In general, the value of $\mathrm{D}$ decreased with the increment of $\mathrm{pH}$, increase of shaking and decrease of temperature.
\end{abstract}

Keywords: humic acids; fractals; turbidimetry.

\section{INTRODUÇÃO}

Dentre as ferramentas usadas para descrever a estrutura ramificada ou a superfície rugosa e distorcida de ácidos húmicos (AH), a teoria fractal aparece como promissora para explicar as mudanças conformacionais de agregados moleculares destas partículas húmicas. A interação dos AH com as superfícies dos minerais, compostos orgânicos, raízes de plantas e microorganismos do solo é determinada por características destes como forma no estado sólido e no estado coloidal, ocorrência de complexos de agregação e fenômeno de dispersão em meio aquoso e pelos vários graus de irregularidade e rugosidade das superfícies expostas ${ }^{1}$. O estudo dessas características desperta grande interesse por parte de pesquisadores em Química Ambiental e Ciências do solo. Pesquisas já têm demonstrado que os AH podem ser descritos como sistemas fractais, tanto no estado sólido ou dissolvido ${ }^{2}$, quanto no coloidal ou em suspensão ${ }^{3}$. Isto implica que moléculas húmicas podem ser decompostas em partes, em que cada uma é "cópia" do "todo", encurtando diferentes fatores em diferentes direções ${ }^{4}$.

O termo "fractal" foi introduzido na década de 70 pelo matemático Benôit Mandelbrot para designar objetos e estruturas complexas dotadas da propriedade de autossimilaridade 5 . Estruturas autossimilares possuem detalhes (ramificações, poros ou rugosidades) em uma certa faixa de escala de comprimento, cuja forma é a mesma (estatisticamente) em cada escala de observação nessa faixa. Assim, se uma parte da estrutura for ampliada terá a mesma forma do todo ${ }^{4}$.

$\mathrm{O}$ aspecto mais básico de um objeto fractal talvez seja a sua dimensão. Um modo simples e intuitivo de associar uma dimensão a um dado conjunto é contar o número mínimo $\mathrm{N}(\varepsilon)$ de quadrículas, de lado $(\varepsilon)$, necessárias para cobrir completamente o conjunto. Esse número obedece a uma lei de potência: $\mathrm{N}(\varepsilon)=\mathrm{A} \cdot \varepsilon^{-\mathrm{D}}$, que define $\mathrm{e}$ permite calcular o expoente $\mathrm{D}$, que é a dimensão de cobertura ou mais simplesmente, a dimensão fractal ${ }^{6}$.

A dimensão fractal (D) pode apresentar, ao contrário da dimensão euclidiana (d), valores fracionários que dependem do sistema: $1<\mathrm{D}<2$ para uma curva, $2<\mathrm{D}<3$ para uma superfície e $2<\mathrm{D}<3$ para um 'sólido'. Esse valor é uma medida da proporção do espaço real-

*e-mail: cesar@ufv.br mente ocupado por um sistema desordenado, podendo refletir a conformação das partículas que o constituem ${ }^{7}$.

Medidas de turbidimetria ${ }^{3}$, espalhamento de neutrons ${ }^{2}$, espalhamento de raios- $\mathrm{x}^{8}$, dentre outras, já foram aplicadas no estudo da conformação e fenômenos de agregação de partículas de $\mathrm{AH}$.

A lei de potência: $\tau \propto \lambda^{\beta}$ (em que $\tau$ é a turbidez, $\lambda$ é o comprimento de onda e $\beta$ é um expoente diretamente relacionado com D) é observada em sistemas de partículas em suspensão diluída, conforme demonstrado por meio de um modelo matemático descrito por Horne ${ }^{9}$. A caracterização de $\mathrm{AH}$ como fractal de massa $\left(\mathrm{D}_{\mathrm{m}}\right.$, sistema cuja massa é proporcional à área superficial) ou de superfície $\left(\mathrm{D}_{\mathrm{s}}\right.$, sistema que consiste de duas regiões não fractais separadas por uma superfície fractal) é realizada por meio do expoente $\beta$, a partir da inclinação da parte linear dos pontos do gráfico $\log \tau$ vs $\log \lambda$. Se $\beta<3$, então o AH será um fractal de massa, neste caso $\mathrm{D}_{\mathrm{m}}=\beta$; se $3<\beta \leq 4$, então o AH será um fractal de superfície, neste caso $D_{\mathrm{s}}=6-\beta^{1}$.

Do ponto de vista experimental a turbidimetria $(\tau)$ baseada no monitoramento da luz transmitida (não espalhada e não absorvida) apresenta-se, em comparação com as demais técnicas, como uma boa alternativa no estudo de fractais em suspensões diluídas ${ }^{9}$. A determinação do valor D de $\mathrm{AH}$ pode fornecer informações que indiquem se estas moléculas exibem ou não um comportamento fractal. A presença deste comportamento indicará que mudanças na conformação de AH refletirão em mudanças nos seus valores D. Moléculas enoveladas tendem a apresentar valores de D menores, em relação àquelas que exibem conformação mais expandida. Com isto pode-se inferir sobre os mecanismos de interação destes ácidos (AH) com metais e pesticidas pois, em geral, durante a complexação/adsorção ocorre um "fechamento" da molécula e com isto uma redução no valor D. Neste sentido, pretende-se, por meio de turbidimetria, verificar a variação nos valores de D de partículas húmicas em suspensão aquosa e diluída, em função das condições experimentais como temperatura e velocidade na agitação da suspensão.

\section{MATERIAL E MÉTODOS}

Foram utilizadas duas amostras de $\mathrm{AH}$ (solo e comercial). Os AH extraídos de solo (AH-solo) foram obtidos de uma amostra de Espodossolo (camada $0-50 \mathrm{~cm}$ ) coletado em Lavras Novas-MG. O 
procedimento de extração foi conduzido seguindo as normas da Sociedade Internacional de Substâncias Húmicas ${ }^{10}$ (IHSS). A purificação foi realizada somente por meio de lavagens sucessivas com solução de HF a 5\%, liofilizados e estocados. Os AH-comercial, foram adquiridos da Fukla S.A, sendo as amostras usadas sem purificação.

Os conteúdos de carbono, hidrogênio e nitrogênio nas amostras foram determinados via analisador de elementos CNHS (Perkin Elmer, PE-2400). O teor de cinzas foi determinado por calcinação, segundo Piccolo ${ }^{11}$. Os espectros de RMN ${ }^{13} \mathrm{C}$ das amostras, no estado sólido, foram realizados em espectrômetro Varian (Unity 400).

Suspensões diluídas $\left(30 \mathrm{mg} \mathrm{L}^{-1}\right)$ das duas amostras de $\mathrm{AH}$ foram preparadas em água deionizada. Para evitar o efeito do espalhamento múltiplo e interação entre partículas o uso de suspensões diluídas é requerido. $\mathrm{O} \mathrm{pH}$ das suspensões foi ajustado para 3,0; 5,0 e 7,0 pela adição de $\mathrm{NaOH}\left(0,1 \mathrm{~mol} \mathrm{~L}^{-1}\right.$ ou $\left.0,001 \mathrm{~mol} \mathrm{~L}^{-1}\right)$ ou $\mathrm{HCl}\left(0,1 \mathrm{~mol} \mathrm{~L}^{-1}\right.$ ou $0,001 \mathrm{~mol} \mathrm{~L}^{-1}$ ), na mesma força iônica. As suspensões foram agitadas durante $24 \mathrm{~h}$ por meio de agitação magnética e horizontal à temperatura ambiente e a $5{ }^{\circ} \mathrm{C}$.

As medidas da turbidez $(\tau)$ nas suspensões de $\mathrm{AH}$ foram realizadas em espectrofotometro UV-Vis (Hitachi U2000), utilizando-se célula de quartzo de um centímetro de percurso óptico. As medidas de ' $\tau$ ' em função de ' $\lambda$ ' foram obtidas na faixa de 400 a $550 \mathrm{~nm}$ a intervalos de $1 \mathrm{~nm}$ e para obtenção de $\beta$ (coeficiente angular) um modelo de equação linear foi ajustado aos pontos do gráfico $\log \tau \nu s$ $\log \lambda$, usando os valores de comprimentos de onda $\lambda$ em metros.

\section{RESULTADOS E DISCUSSÃO}

Os conteúdos de carbono, hidrogênio e nitrogênio e teor de cinzas são apresentados na Tabela 1 . A razão N/C para AH-solo igual a 0,034 está coerente com as obtidas por Spark et al. ${ }^{12}$ que caracterizou ácidos húmicos extraídos de solos. $\mathrm{O}$ valor encontrado para $\mathrm{AH}-$ comercial igual a 0,016 mostra que o material não é extraído de solo. $\mathrm{O}$ teor de cinzas para o AH-comercial foi maior, pois este foi utilizado sem prévia purificação. A análise de $\mathrm{RMN}{ }^{13} \mathrm{C}$ para $\mathrm{AH}$-solo mostrou sinais de carbono pertencentes aos grupos alifáticos (0-39 ppm de alta intensidade), de aromáticos (106-165 ppm de alta) e carboxílicos (170-190 ppm de baixa). Para o AH-comercial a análise de RMN sugere apenas os grupos alifáticos (0-39 ppm de alta intensidade). Estes resultados mostram que o $\mathrm{AH}$-solo apresenta constituintes mais aromáticos, enquanto que $\mathrm{AH}$-comercial apresenta praticamente apenas constituintes alifáticos.

Tabela 1. Análise elementar e teor de cinzas dos AH

\begin{tabular}{lcccc}
\hline Amostras & $\mathrm{C}$ & $\mathrm{H}$ & $\mathrm{N}$ & Cinzas \\
\cline { 2 - 5 } & \multicolumn{5}{c}{$(\%)$} \\
\hline AH-solo & 50,8 & 3,51 & 1,75 & 8 \\
AH-comercial & 43,1 & 4,87 & 0,67 & 14 \\
\hline
\end{tabular}

A dependência em lei de potência de $\tau$ em função de $\lambda$ foi confirmada para todas as suspensões nas diferentes condições, visto que os ajustes lineares aos pontos experimentais do gráfico do $\log \tau$ em função do $\log \lambda(\mathrm{m})$ apresentaram coeficiente de determinação $\mathrm{R}>0,99$. Assim, as partículas em estudo podem exibir estrutura fractal de massa $\left(D_{m}\right)$ ou de superfície $\left(D_{s}\right)$, e estes valores $\left(D_{m}\right.$ e $\left.D_{s}\right)$ podem ser obtidos diretamente do coeficiente angular $(\beta)^{1}$. A Figura 1 mostra este comportamento para $\mathrm{AH}$-solo, a temperatura de $25^{\circ} \mathrm{C}$ e agitação horizontal, em diferentes valores de $\mathrm{pH}$. Neste caso os valores dos coeficientes angulares $\beta$ foram 2,73; 3,14 e 3,14 em pH 3,0; 5,0 e 7,0, respectivamente, fornecendo os valores de $\mathrm{D}$ em cada condição experimental $\left(\mathrm{D}_{\mathrm{m}}=2,73, \mathrm{D}_{\mathrm{s}}=2,86\right.$ e $\left.\mathrm{D}_{\mathrm{s}}=2,86\right)$.

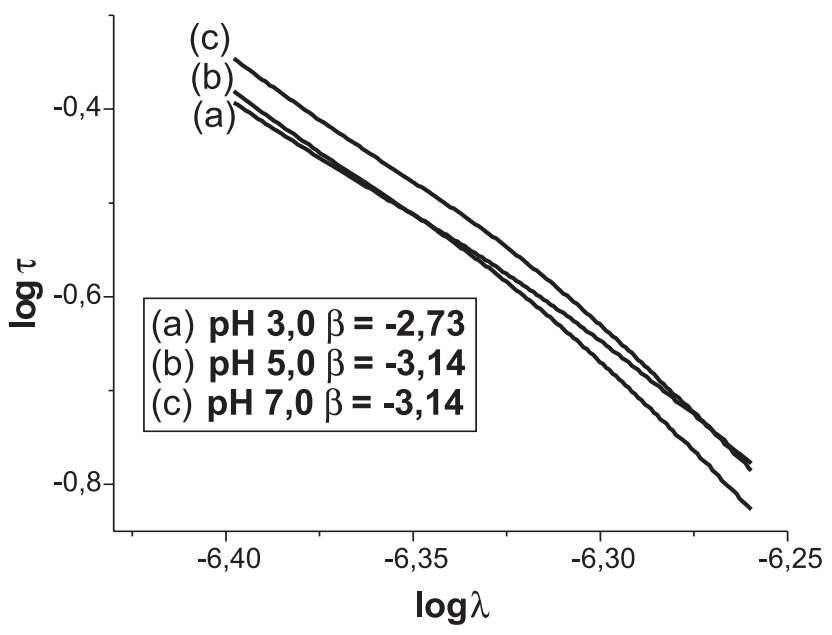

Figura 1. Obtenção dos valores de $\beta$ para as suspensões aquosas de AHsolo, em diferentes valores de $\mathrm{pH}$, a temperatura ambiente e sob agitação horizontal

A Tabela-2 apresenta um resumo das dimensões fractais D dos AH (solo e comercial), em diferentes condições experimentais. Comparando os valores de D nas duas amostras observa-se que estes valores são maiores para $\mathrm{AH}$-solo, exceto em pH 3,0. Estes resultados estão coerentes com a análise por RMN que sugere estruturas com predominância de constituintes aromáticos (AH-solo) que são mais fechadas o que implica em um maior valor de D, quando comparadas com as alifáticas que estão presentes em maiores quantidades no AH-comercial. Observa-se ainda que o AH-solo sofre uma transição morfológica em sua estrutura passando de fractal de massa ( $\mathrm{pH} 3,0)$ para fractal de superfície ( $\mathrm{pH} \geq 5,0)$, fato que não é observado para o AH-comercial. Essa transição sugere que as partículas húmicas do solo, em suspensão, evoluíram de estruturas ramificadas, fragmentadas e menos compactas para estruturas compactas com superfícies irregulares ou rugosas com o aumento do $\mathrm{pH}$. Wershaw ${ }^{13}$ reforça que a estrutura molecular de $\mathrm{AH}$, bem como seus mecanismos de agregação, podem sofrer variação em função de sua origem. A diminuição nos valores de ' $\mathrm{D}_{\mathrm{m}}$ ' (AH-solo e AH-comercial a temperatura de $5{ }^{\circ} \mathrm{C}$ e diferentes agitações) indica a passagem de estruturas compactas, de superfície levemente rugosa, para estruturas mais expandidas. Já a diminuição nos valores de ' $\mathrm{D}$ ' '(AH-comercial a temperatura ambiente e diferentes tipos de agitação) sugere a variação na conformação, passando de formas menos alongadas para mais alongadas ${ }^{2}$.

Visando conhecer a contribuição da eficiência da agitação das suspensões na conformação das partículas húmicas, os valores de D foram determinados utilizando agitação horizontal e magnética. $\mathrm{Na}$ agitação magnética o choque entre as partículas é maior que na horizontal, pois se utilizam "barrinhas" que aumentam a intensidade da interação mecânica. Silva e colaboradores ${ }^{14,15}$ verificaram diferenças entre os tipos de agitação (horizontal e magnética) e em diferentes tempos de agitação sobre os valores de D. Analisando os resultados da Tabela 2 em relação aos tipos de agitação, observa-se que os valores de D sofreram uma ligeira diminuição quando as suspensões foram submetidas à agitação magnética, o que pode estar relacionado às intensidades dos choques entre as partículas.

A variação na temperatura das suspensões também provoca alterações no comportamento conformacional de $\mathrm{AH}^{16}$. Verificou-se que 
Tabela 2. Resumo dos valores de D obtidos em diferentes condições experimentais

\begin{tabular}{|c|c|c|c|c|c|}
\hline \multirow[t]{2}{*}{ AMOSTRA } & \multirow[t]{2}{*}{$\mathrm{pH}$} & \multicolumn{2}{|c|}{ Agitação horizontal } & \multicolumn{2}{|c|}{ Agitação magnética } \\
\hline & & $\mathrm{D}\left(25^{\circ} \mathrm{C}\right)$ & $\mathrm{D}\left(5^{\circ} \mathrm{C}\right)$ & $\mathrm{D}\left(25^{\circ} \mathrm{C}\right)$ & $\mathrm{D}\left(5^{\circ} \mathrm{C}\right)$ \\
\hline AH-solo & 3,0 & $\mathrm{D}_{\mathrm{m}}=2,73 \pm 0,01$ & $\mathrm{D}_{\mathrm{m}}=1,94 \pm 0,25$ & $\mathrm{D}_{\mathrm{m}}=2,70 \pm 0,01$ & $\mathrm{D}_{\mathrm{m}}=1,90 \pm 0,23$ \\
\hline AH-solo & 5,0 & $\mathrm{D}_{\mathrm{s}}=2,86 \pm 0,03$ & $\mathrm{D}_{\mathrm{m}}=1,04 \pm 0,19$ & $\mathrm{D}_{\mathrm{s}}=2,84 \pm 0,02$ & $\mathrm{D}_{\mathrm{m}}^{\mathrm{mi}}=1,01 \pm 0,20$ \\
\hline AH-solo & 7,0 & $\mathrm{D}_{\mathrm{s}}=2,86 \pm 0,04$ & $\mathrm{D}_{\mathrm{m}}=1,02 \pm 0,17$ & $\mathrm{D}_{\mathrm{s}}=2,82 \pm 0,04$ & $\mathrm{D}_{\mathrm{m}}=1,00 \pm 0,18$ \\
\hline AH-comercial & 3,0 & $\mathrm{D}_{\mathrm{s}}=2,85 \pm 0,02$ & $\mathrm{D}_{\mathrm{m}}=1,72 \pm 0,15$ & $\mathrm{D}_{\mathrm{s}}=2,83 \pm 0,01$ & $\mathrm{D}_{\mathrm{m}}=1,70 \pm 0,12$ \\
\hline AH-comercial & 5,0 & $\mathrm{D}_{\mathrm{s}}=2,51 \pm 0,05$ & $\mathrm{D}_{\mathrm{m}}=1,06 \pm 0,18$ & $\mathrm{D}_{\mathrm{s}}=2,50 \pm 0,03$ & $\mathrm{D}_{\mathrm{m}}=1,02 \pm 0,15$ \\
\hline AH-comercial & 7,0 & $\mathrm{D}_{\mathrm{s}}=2,51 \pm 0,03$ & $\mathrm{D}_{\mathrm{m}}=1,68 \pm 0,30$ & $D_{s}=2,49 \pm 0,01$ & $\mathrm{D}_{\mathrm{m}}=1,58 \pm 0,28$ \\
\hline
\end{tabular}

Obs. As medidas de D foram obtidas de médias de três repetições.

houve diminuição nos valores de $\mathrm{D}$ das suspensões de $\mathrm{AH}$-solo e AH-comercial, a uma temperatura abaixo da ambiente, sugerindo o predomínio de fractais de massa (Dm). Österberg e Mortensen ${ }^{2}$ também observaram redução nos valores de $\mathrm{D}$ e, com isto, um rearranjo na agregação de partículas húmicas com a diminuição da temperatura de 21 para $8{ }^{\circ} \mathrm{C}$. A presença destes fractais $\left(\mathrm{D}_{\mathrm{m}}\right)$ pode estar relacionada com a fragmentação crescente das estruturas ramificadas quando as amostras são resfriadas. Com a fragmentação, essas partículas seriam menores, quase desprovidas de ramificações e, portanto, poderiam se agregar de uma forma mais compacta favorecendo a formação de fractais de massa com estruturas desordenadas cujas escalas de massa e de superfície são semelhantes.

\section{CONCLUSÕES}

Os valores de $\mathrm{D}$ apresentados neste trabalho indicam que a determinação da dimensão fractal (D) de $\mathrm{AH}$ em suspensões aquosas e diluídas, utilizando método turbidimétrico é viável, mostrando que o valor D aqui obtido foi dependente das condições experimentais. É importante ressaltar que este estudo tem ainda caráter exploratório, portanto, para melhor compreensão da aplicabilidade desta ferramenta em estudos de $\mathrm{AH}$, pesquisas adicionais devem ser feitas visando comparar resultados e fornecer informações sobre aspectos dinâmicos de agregação das partículas de $\mathrm{AH}$, para revelar as características físico-químicas que controlam a natureza fractal dessas partículas. Compreendendo melhor o comportamento das partículas de $\mathrm{AH}$ no ambiente, quanto à sua conformação, podem-se fazer previsões em relação às interações destes com os agentes químicos poluentes.

\section{REFERÊNCIAS}

1. Österberg, R.; Mortensen, K. Em Humic substances in the global environment and implications on human health; Senesi, N.; Miano, T. M., eds.; Elsevier Science: Amsterdam, 1994, p. 810.

2. Senesi, N.; Rizzi, F. R.; Dellino, P.; Acquafredda, P.; Soil Sci. Soc. Am. J. 1996, 60, 1773 .

3. Mandelbrot, B. B.; The fractal geometry of nature - update and augmented, Freeman; New York, 1983.

4. Chaves, C. M. G. F.; Ciência Hoje 1989, 10, 27.

5. Vicsek, T.; Fractal growth fenomena, World Scientic Publishing: Singapore, 1992.

6. Harrison, A.; Fractals in chemistry, Science Publications: Oxford, 1992.

7. Rice, J. A.; Lin, J. S.; Environ. Sci. Technol. 1993, 27, 413.

8. Horne, D. S.; Faraday Discuss. 1987, 83, 259.

9. Senesi, N.; Rizzi, F. R.; Dellino, P.; Acquafredda, P.; Colloids Surf., Part A 1997, 127, 57.

10. Swift, R. S. Em Method for extraction of IHSS soil fulvic and humic acids; Spark, K. M.; Wells, J. D.; Johnson, B. B., eds.; SSSA: Oxford, 1996, p. 1018.

11. Piccolo, A.; Soil Sci. 1988, 146, 418

12. Spark, K. M.; Wells, J. D.; Johnson, B. B.; Aust. J. Soil Res. 1997, 35, 89.

13. Wershaw, R. L.; Soil Sci. 1999, 164, 803.

14. Silva, A. C.; Mendonça, E.; Revista Ceres 2000, 269, 103

15. Silva, A. C.; Mendonça, E; Lobato, M.; Reis, C.; Rev. Bras. Ci. Solo 2000, 24,759 .

16. Stevenson, J. F.; Humus chemistry - Genesis, composition, reactions, John Wiley \& Sons: New York, 1994. 